\title{
SOFTWARE PARA LA PROGRAMACIÓN DE UN RIEGO DE PRECISIÓN EN EL CULTIVO DE LA FRESA
}

\author{
González Perea, R. ${ }^{1}$, Fernández García, I. ${ }^{1}$, Martin Arroyo, $M .{ }^{1}$, Rodríguez Díaz J.A. ${ }^{1}$, \\ Camacho Poyato, E. ${ }^{1}$, Montesinos, P. ${ }^{1}$
}

${ }^{1}$ Departamento de Agronomía. Universidad de Córdoba. Campus Rabanales, Edif. Da Vinci, 14071. Córdoba.E-mail: g72goper@uco.es,g52fegai@uco.es,o02maarm@uco.es, jarodriguez@uco.es, pmontesinos@uco.es, ecamacho@uco.es

\section{Resumen}

Bajo un escenario de menor disponibilidad de agua, aumento de la frecuencia de las sequías y las incertidumbres asociadas al cambio climático, el sector del riego en Europa tiene que aumentar la productividad del agua mediante una mejora de la eficiencia en el uso de la misma. El sector fresero concentrado en un entorno de alta sensibilidad medioambiental (Parque Nacional de Doñana, Huelva, España) es un buen ejemplo de cultivo con alta dependencia de recursos hídricos. Más del $90 \%$ de la producción es destinada a mercados internacionales que demandan fresas de alta calidad, producidas en condiciones sostenibles. Por tanto, para asegurar la supervivencia del cultivo de fresa en la zona de Huelva resulta imprescindible desarrollar herramientas que permitan a los productores un uso óptimo de estos recursos hídricos. Por ello, el objetivo de este trabajo fue desarrollar un software (Irri-Fresa) que permita a los productores de fresa en la zona de Huelva realizar una programación de riego que maximice la eficiencia en el uso de los recursos hídricos, teniendo en cuenta la configuración hidráulica de cada sector de riego así como las condiciones climáticas en tiempo real de la zona y el estado fenológico del cultivo. Irri-Fresa proporciona al sector del cultivo de la fresa una herramienta fácil y rápida para obtener una programación de riego óptima y actualizada por semanas y personalizada por cada usuario. Este software, actualmente, está siendo usado satisfactoriamente por diversos productores de la zona, obteniendo ahorros significativos de agua, ayudando a concienciar al sector fresero de la importancia de conocer y optimizar sus consumos de agua. 


\section{1- Introducción}

La escasez de agua y la creciente preocupación sobre el impacto que el uso excesivo de esta agua puede ocasionar en el medio ambiente hace que la agricultura de regadío se encuentre amenazada (Fereres et al., 2011). En un escenario de menor disponibilidad de agua, aumento de la frecuencia de las sequías y las incertidumbres asociadas al cambio climático, el sector del riego en Europa tiene que aumentar la productividad del agua mediante una mejora de la eficiencia en el uso de la misma (Hunt, 2004; Rodríguez Díaz et al., 2007). La producción de fresas es un buen ejemplo de cultivo intensivo de regadío que está muy demandado por los mercados europeos tanto para consumo en fresco como para industria. La mayor concentración de la producción de fresas en Europa y la segunda en el mundo se encuentra en Huelva (Sureste de España). Más del $90 \%$ de la producción es destinada a mercados internacionales que demandan fresas de alta calidad, producidas en condiciones sostenibles. El $73 \%$ de la producción total de fresa en Huelva se encuentra en las inmediaciones del Parque Nacional de Doñana, área con una alta sensibilidad medioambiental (Fundación Doñana 21, 2006). Esto unido a la alta dependencia que este cultivo tiene de los recursos hídricos hace que exista una gran preocupación por la sostenibilidad ambiental de la producción del cultivo en la zona. Por tanto, un uso óptimo de este recurso resulta fundamental para asegurar la supervivencia del cultivo de fresa en la zona de Huelva.

El establecimiento de un riego de precisión óptimo, que aporte en cada momento el agua necesaria según las condiciones climáticas y estado fenológico de la planta, es de vital importancia para conseguir un uso óptimo del recurso hídrico. Un riego de precisión implica tanto la evaluación precisa de las necesidades de agua de los cultivos como la aplicación exacta de agua en el momento y el lugar adecuado y el uso de elementos hidráulicos con altas eficiencias volumétricas que permitan aplicación espacialmente uniformes (Smith et al., 2010). Los sistemas de micro-riego son capaces de aplicar pequeñas cantidades de agua en intervalos de tiempo cortos. Estos sistemas se diseñan para mantener la zona del suelo donde se encuentra la mayor densidad radicular cerca de un nivel óptimo de humedad. Estos sistemas se pueden controlar fácilmente y normalmente, son automatizados (Phene and Howell, 1984; Meron et al., 1996; Dukes and Scholberg, 2004; Wanjura et al., 2004; Evett et al., 2006).

Son muchos los autores que han desarrollado metodologías para realizar una programación de riego que maximice el uso del agua. Kloke et al., 2009, por ejemplo, establece una herramienta para realizar una programación basándose en un riego deficitario. El alto valor económico que posee el sector fresero junto con las estrategias comerciales que los agricultores persiguen hace que el riego deficitario no sea una práctica viable en este entorno. Otros autores han desarrollado metodologías y herramientas de programación de riego, para otros cultivos, basándose en balances de agua en el suelo, o restricciones de agua en un determinado periodo (Bergez et al., 2000; Bartlett et al., 2014) sin tener en cuenta la configuración del sector de riego, la eficiencia del sistema hidráulico o la uniformidad de distribución del agua en parcela. La eficiencia de un sistema de micro riego, principalmente el riego por goteo, habitualmente es considerada como más del $90 \%$. Sin embargo, como en otros sistemas, la capacidad de lograr un alto nivel de eficiencia es más una función de gestión que una propiedad intrínseca del sistema (Smith et al., 2010). Por ejemplo, Shannon et al. (1996) encontraron que la eficiencia de la aplicación de un riego por goteo en condiciones comerciales en un cultivo de caña de azúcar varió entre 30 y el $90 \%$, debido principalmente, a un exceso de riego y percolación profunda. Las principales causas por las que un sistema de riego puede presentar una baja uniformidad se centran en la variación de presión a lo largo de un ramal de riego, la obstrucción de emisores y/o una gestión no adecuada del riego.

Todo esto, unido a las particulares condiciones en las que se desarrolla el cultivo de fresa en Huelva (suelos extremadamente arenosos, uso de cintas de riego no adecuadas 
para realizar un riego de precisión, alta variabilidad en la configuración de sectores de riego, desarrollo del cultivo tanto fuera como dentro de macrotúneles, etc.) hace que establecer una programación de riego óptima sea una tarea extremadamente compleja. Por ello, el objetivo de este trabajo es desarrollar un software que permita a los productores de fresa en la zona de Huelva realizar una programación de riego que maximice la eficiencia en el uso de los recursos hídricos, teniendo en cuenta la configuración hidráulica de cada sector de riego así como las condiciones climáticas en tiempo real de la zona y el estado fenológico del cultivo.

\section{2- Metodología}

\subsection{Programación del riego}

Para realizar una óptima programación de riego en el cultivo de la fresa son muchos los factores que deben ser considerados, debido a sus condiciones particulares de producción. Necesidades teóricas del cultivo, suelos muy arenosos, producción bajo plástico, alta variabilidad de configuración de sectores, etc. son algunos de estos factores que influyen en el cálculo del tiempo total de riego.

Para el cálculo de las necesidades teóricas del cultivo $\left(E T_{c}\right)$ se empleó la ecuación desarrollada por Allen et al. (1998) adaptada para considerar las condiciones particulares de producción de fresa (Ec. 1):

$$
E T_{c}=\sum_{\substack{i=1 \cdots n \\ j=1 \cdots n}} K_{c_{i j}} \cdot E T_{o_{f p}}+\sum_{\substack{i=n+1 \cdots k \\ j=m+1 \cdots l}} K_{c_{i j}} \cdot E T_{o_{b p}}
$$

donde $i$ es el índice que indica el día de la campaña, $j$ es índice que indica la fase de desarrollo en la que se encuentra el cultivo, $\mathrm{K}_{\mathrm{c}}$ es el coeficiente de cultivo que varía a lo largo del ciclo de producción y cuyos valores se obtuvieron de trabajos previos en la zona (Allen et al., 2006 and WWF, 2009), $\mathrm{ET}_{\text {o fp }}$ y $\mathrm{ET}_{\mathrm{o} \mathrm{bp}}\left(\mathrm{mm} \mathrm{dia}^{-1}\right)$ son la evapotranspiración de referencia estimada fuera y dentro del macrotúnel, respectivamente. $\mathrm{ET}_{\mathrm{o}}$ fp se obtuvo a través del promedio de una serie histórica de los últimos 10 años de las estaciones agroclimáticas más cercanas a la zona de estudio y $\mathrm{ET}_{\mathrm{o}}$ bp se obtuvo corrigiendo la serie histórica de $\mathrm{ET}_{\mathrm{ofp}}$ con un valor de transmisividad de un plástico tipo utilizado en la zona para cubrir los macrotúneles.

Por otro lado, las características del sector de riego (tipo de emisor, número de macrotúneles, dimensiones de los mismos, etc.) tienen una gran influencia sobre la eficiencia en la aplicación del agua de riego (rendimiento de aplicación, $R_{a}$ ). $R_{a}$ es un índice relacionado con la uniformidad de un sector de riego $\left(U_{\text {sr }}\right)$ (Martín, 2013; Bralts and Kesner, 1983) que se define como la relación entre el volumen de agua aplicada por los emisores de riego y el volumen de agua aprovechado por la planta. Así, este índice transforma las necesidades de riego teóricas del cultivo $\left(\mathrm{ET}_{\mathrm{c}}\right)$ en las necesidades de agua brutas que se requiere para satisfacer las necesidades de riego de los cultivos. Mediante el software de simulación hidráulica EPANET (Rossman, 2002) se obtuvo una relación entre $\mathrm{R}_{\mathrm{a}}$ y la superficie del sector de riego, para las configuraciones típicas de la zona. Así, en la Tabla 1 se representa los valores de $R_{a}$ obtenidos para cada tamaño de sector de riego.

Tabla 1. Relación entre $R_{a}$ y superficie de un sector de riego.

\begin{tabular}{lcccc}
\hline & \multicolumn{5}{c}{ Superficie del sector $\left(\mathbf{S}_{\text {sector }}\right)($ ha) } \\
\cline { 2 - 6 } & $\mathrm{S}_{\text {sector }} \leq 0.25$ & $0.25<\mathrm{S}_{\text {sector }} \leq 0.5$ & $0.5<\mathrm{S}_{\text {sector }} \leq 0.75$ & $\mathrm{~S}_{\text {sector }}>0.75$ \\
\hline $\mathbf{R}_{\mathbf{a}}$ & 0.9 & 0.85 & 0.8 & 0.75 \\
\hline
\end{tabular}


Los tipos de cintas de riego utilizados en la zona difieren básicamente en el caudal aportado por las mismas, siendo las más usuales del tipo T-Tape de $3.0,3.8$ y $5.0 \mathrm{I} \mathrm{h}^{-1} \mathrm{~m}^{-1}$. El tiempo de llenado de los ramales de riego se ve afectado tanto por el tipo de emisor como por las características geométricas de los macrotúneles. El efecto del tiempo de llenado de la cinta de riego fue considerado en el cálculo de la programación del riego.

\subsection{Software.}

El software desarrollado (Irri-Fresa) consta de dos módulos, ambos desarrollados con el lenguaje de programación multiplataforma JAVA.

El primer módulo es un software para PC en el cuál el usuario caracteriza la ubicación de la finca, fecha de plantación, fecha de cubrición con plástico, número de macrotúneles, número de lomos y dimensiones de los macrotúneles, caudal del emisor y número de electroválvulas para todos y cada uno de sus sectores de riego de una o más fincas. Posteriormente, el usuario puede elegir entre diferentes opciones de programación con distintos intervalos temporales. Todo ello se conoce como los inputs del programa. La salida u output de este módulo es una programación del riego (en minutos por día) para toda la campaña basándose en el promedio de los datos climáticos de una serie histórica de los 10 últimos años. Posteriormente, dicha programación se actualiza en tiempo real con los datos meteorológicos reales de la campaña. En la Figura 1 se muestra el esquema operacional de Irri-Fresa para PC.

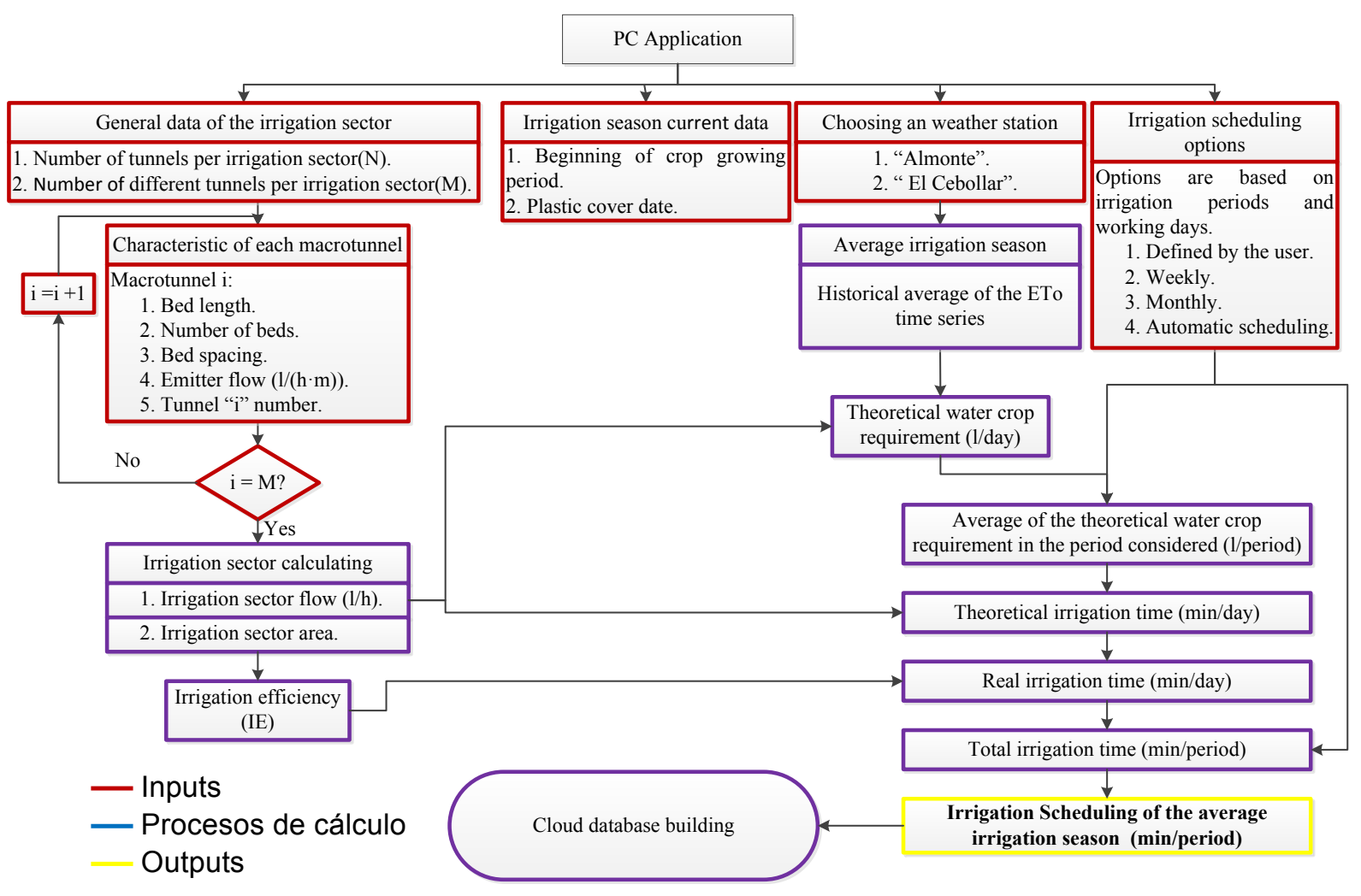

Figura 1. Esquema operacional de Irri-Fresa para PC.

La programación de riego obtenida (output) es almacenada en una base de datos creada mediante el lenguaje de programación MySQL y colocada en una nube virtual. Esta base consta de cinco tablas (usuarios, fincas, sectores, programación de riego estimada, programación de riego real) en las que se recogen los usuarios del programa, características de sus fincas y sectores y tanto la programación estimada de toda la campaña (output del 
software para PC) como la programación de riego real actualizada con las estaciones climáticas más cercanas a la zona.

El segundo módulo se basa en una aplicación móvil (APP) en forma de widget disponible para cualquier dispositivo que soporte Android. Dicha aplicación está conectada en tiempo real con las estaciones agroclimáticas más cercanas a la zona con el objetivo de corregir con los datos climáticos en tiempo real, la programación de riego previamente realizada (output del software para PC). Así, con esta APP el agricultor dispondrá para cada semana de la programación de riego de cada una de sus fincas. En la Figura 2 se muestra el esquema operacional de la APP de Irri-Fresa.

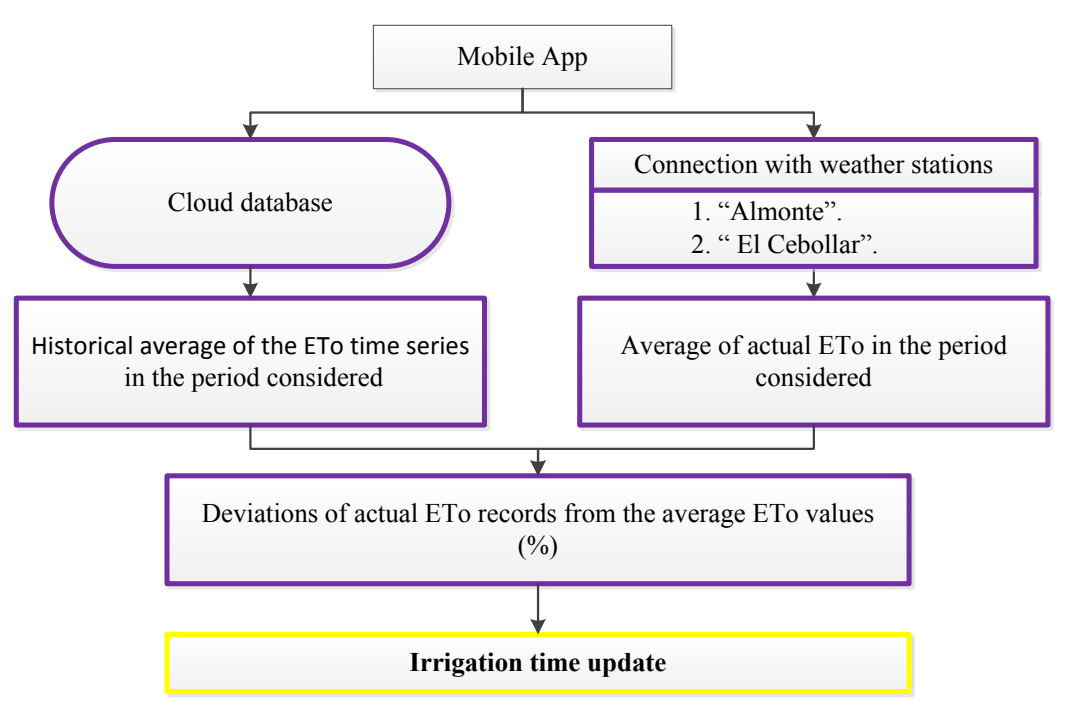

Figura 2. Esquema operacional de la APP de Irri-Fresa.

\section{3- Resultados y discusión}

El software desarrollado para PC consta de 12 ventanas. En la Figura 3 se muestran las dos primeras ventanas $(1,2)$, que se corresponden con la ventana de bienvenida al programa y de identificación del usuario.
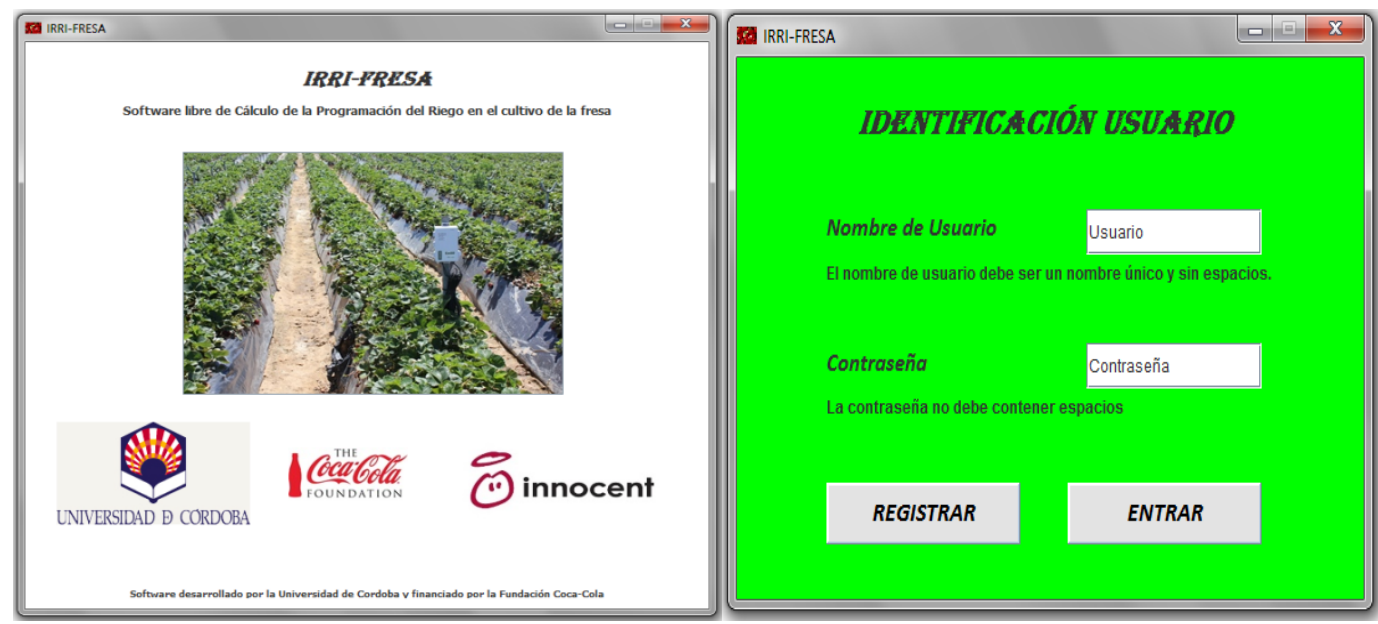

Figura 3. Ventana de bienvenida (izquierda) y ventana de identificación (derecha). 
Las tres siguientes pantallas $(3,4,5)$ están destinadas a la configuración de una finca y un sector (nombre de la finca, ubicación, nombre del sector, configuración del sector etc.). En la figura 4 se muestra la ventana de identificación de la finca (pantalla 3).

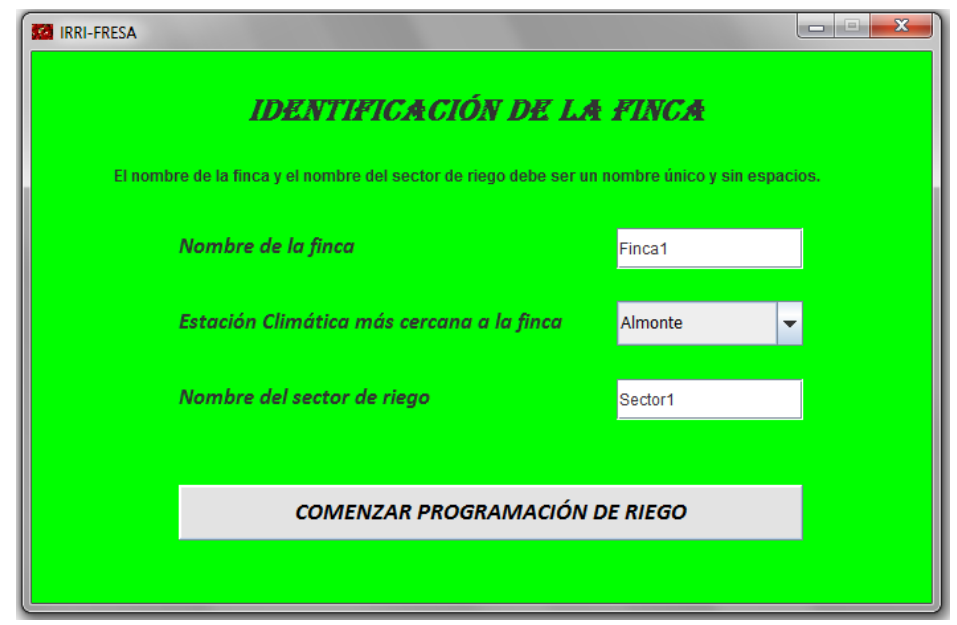

Figura 4. Ventana de identificación de la finca.

En la pantalla número 6 se caracteriza la campaña en la cual se quiere realizar la programación. En ella se establece la fecha estimada de inicio de riego con cinta y la fecha estimada de cubrición de los macrotúneles con plástico.

Las ventanas $7,8,9,10$ y 11 es donde se realiza la programación de riego propiamente dicha. El usuario podrá elegir entre 4 tipos de programaciones distintas, desde la más personalizada donde el usuario establece los intervalos de tiempo por meses y los días de la semana que desea regar en cada uno de esos intervalos (Personalizado), hasta una programación recomendada (Recomendado), donde el usuario únicamente decidirá los días de la semana que desea regar en los intervalos automáticamente establecidos. En la figura 5 se muestra el menú de selección de los distintos tipos de programación que IrriFresa proporciona.

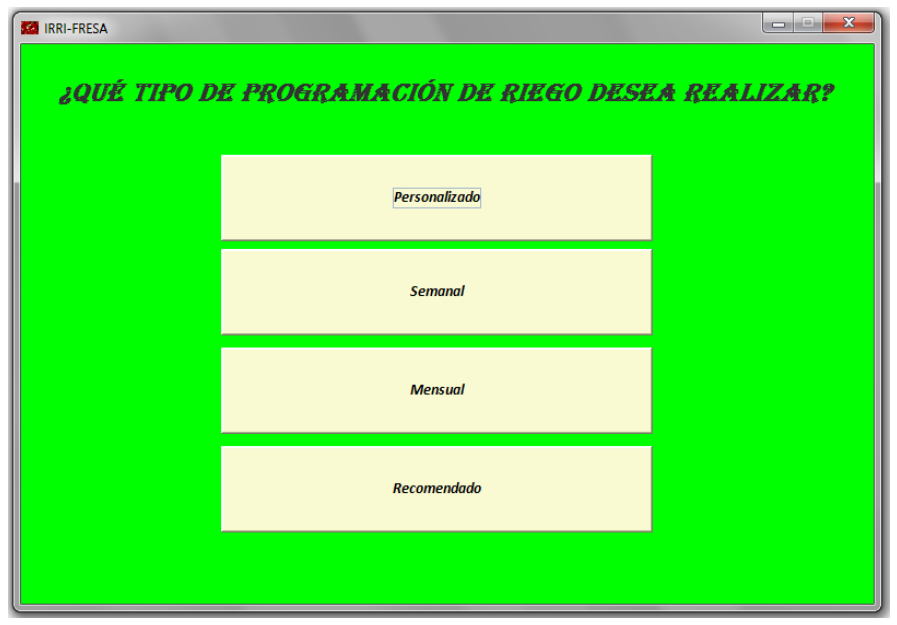

Figura 5. Menú de selección del tipo de programación.

La última ventana (número 12) permite descargar la programación realiza tanto en formato PDF como en formato $x / s$ o xlsx (Excel). En la figura 6 se muestra la última ventana y un ejemplo de salida en PDF. 


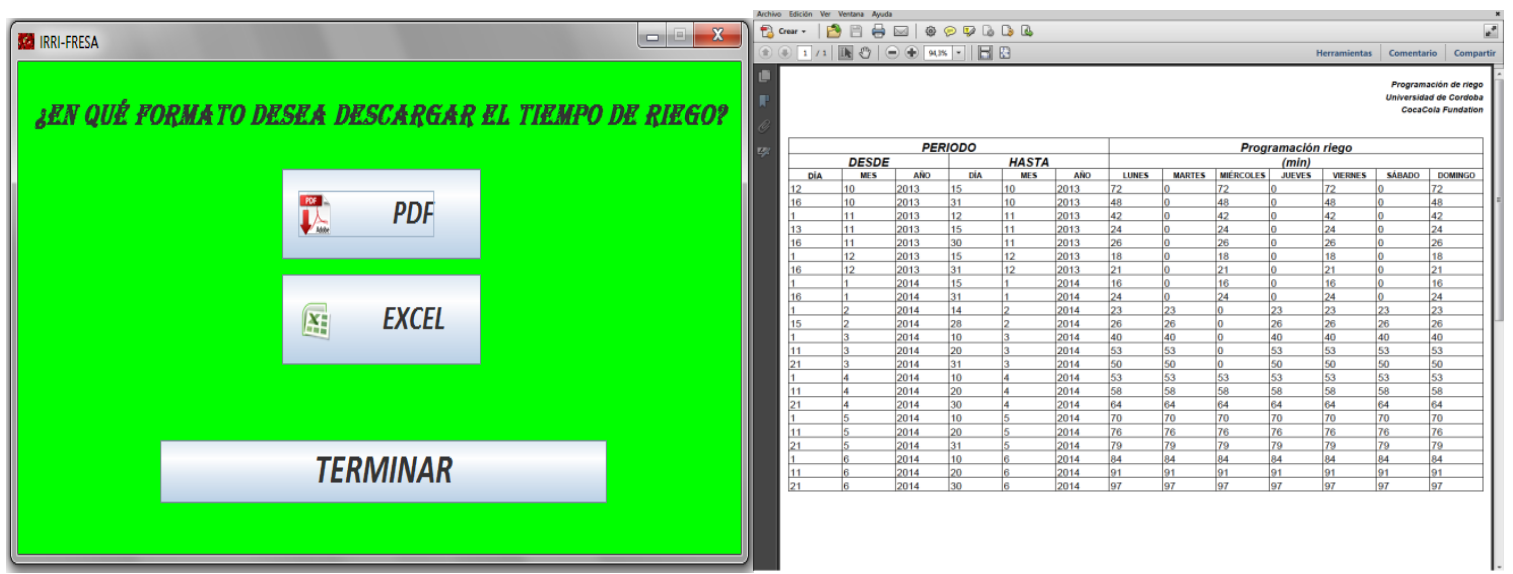

Figura 6. Ventana de descarga de programación (izquierda) y ejemplo de una salida del programa en PDF (derecha).

Durante la campaña 2014/2015, el software desarrollado está en versión beta y está siendo usada por diferentes agricultores de la zona.

En la figura 7 se muestra el widget correspondiente a la APP de Irri-Fresa. En ella se puede ver la programación de la semana actual corregida en tiempo real con los datos climáticos de la zona donde se sitúa cada sector. Debido a que al final de campaña la cubierta de los macrotúneles donde se produce la fresa es retirada pero se sigue regando con la cinta de riego, el widget cuenta también con la opción de retirar o poner dicho plástico de los macrotúneles modificando así los tiempos de riego. Cuando el tiempo de riego excede un determinado valor, se recomienda al agricultor dividir ese tiempo de riego en más de un pulso. El widget cuenta también con una lista desplegable para poder ver la programación que el agricultor ha realizado en los distintos sectores y fincas. 


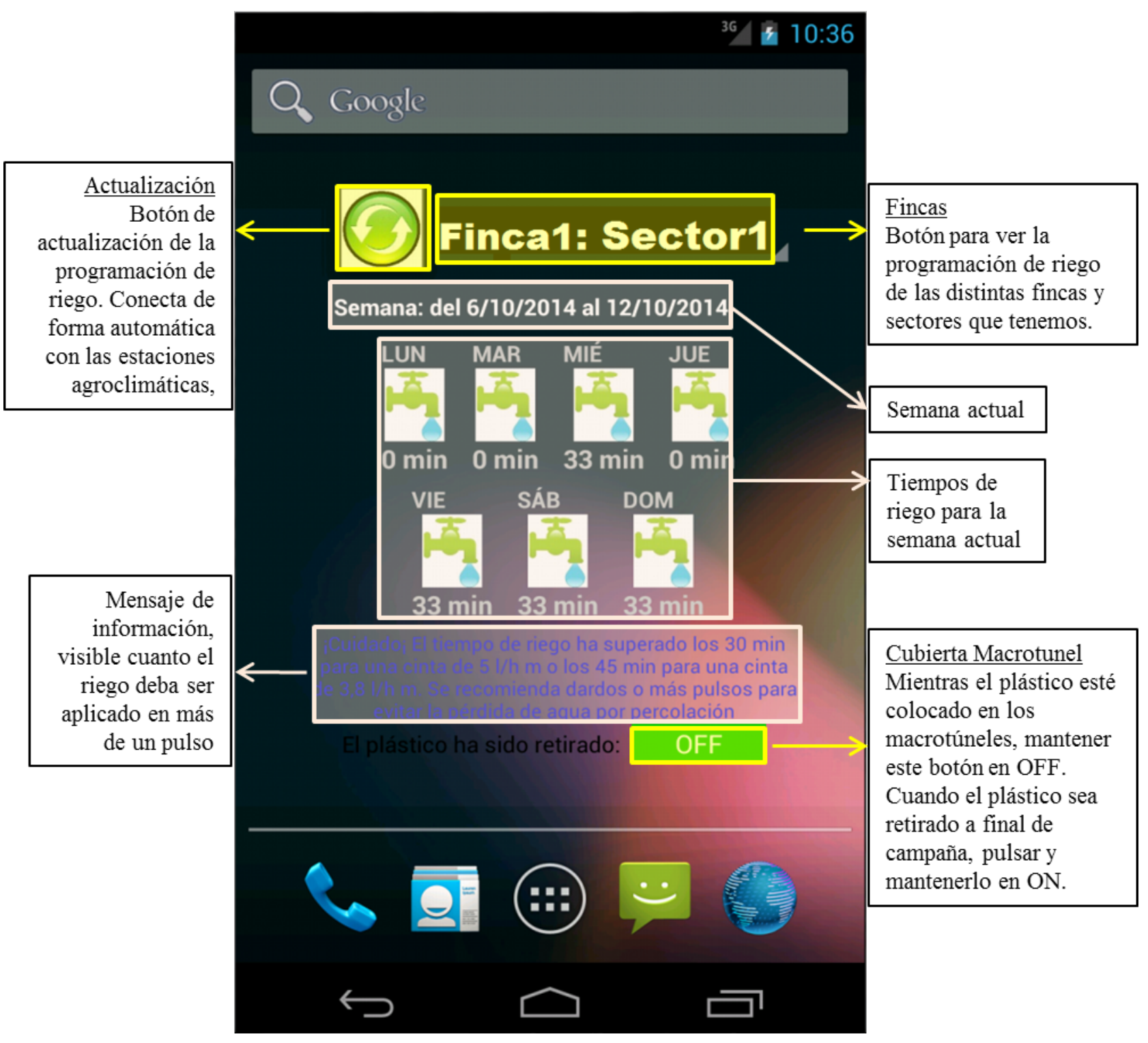

Figura 7. APP de Irri-Fresa.

\section{4- Conclusiones}

Irri-Fresa proporciona al sector del cultivo de la fresa una herramienta fácil y rápida para obtener una programación de riego óptima y actualizada por semanas y personalizada por cada usuario. Esta aplicación, actualmente, está siendo usada satisfactoriamente por diversos productores de la zona, obteniendo ahorros significativos de agua, ayudando a concienciar al sector fresero de la importancia de conocer y optimizar sus consumos de agua.

\section{5- Bibliografía}

Allen, R. G.; Pereira, L. S.; Raes D.; Smith, M. (2006). "Guías para la determinación de los requerimientos de agua de los cultivos". Estudio FAO Riego y drenaje 56. 
Bartlett, A.C., Andales, A.A., Arabi, M., Bauder, T.A., 2014. A smartphone app to extend use of a cloud-based irrigation scheduling tool. Computer and electronics in Agriculture. $111,127-130$.

Bergez, J.E., Debaeke, P., Deumier, J.M., Lacroix, B., Leenhardt, D., Leroy, P., Wallach, D., 2001. MODERATO: an object-oriented decision tool for designing maize irrigation schedules. Ecol. Model, 137 (1), 43-60.

Bralts, V.F. and Kesner, C.D. 1983. Drip irrigation Field Uniformity Estimation. Transactions of the Amer. Soc. Ag. Eng. 26(5):1369-1374.

Dukes, M.D., Scholberg, J.M., 2004. Automated subsurface drip irrigation based onsoil moisture. In: ASAE Paper No. 052188.

Evett, S.R., Peters, R.T., Howell, T.A., 2006. Controlling water use efficiency withirrigation aumotation: cases from drip and center pivot irrigation of corn andsoybean. In: Southern Conservation Systems Conference, Amarillo, TX.

Fereres, E., Orgaz, F., Gonzalez-Dugo, V., 2011. Reflections on food security underwater scarcity. J. Exp. Bot. 62, 4079-4086.

Fundación Doñana 21, 2006. Manual de Buenas Prácticas Agrarias Sostenibles.In: De la Agricultura al desarrollo Rural Sostenible, Available at http://www.donana.es/wp content/uploads/2013/01/Manual-Buenas-practicas-agrarias-sostenibles.pdf.

Hunt, C.E., 2004. Thirsty Planet e Strategies for Sustainable Water Management.Zed Books, New York.

Klocke, N., Stone, L., Bolton, D., 2009. Irrigation scheduling for deficit irrigation. World Environ. Water Res. Cong. 2009, 1-9.

Martín Arroyo, M., 2013. Influencia del Riego en la Sostenibilidad del Uso del Agua en el Cultivo de la Fresa en el Entorno del Parque Nacional de Doñana. (Influence of irrigation on water use sustainability in strawberry cultivation in the surroundings of Doñana National Park). Degree dissertation. UCO.

Meron, M.R., Hallel, R., Shay, G., Feuer, R., 1996. Soil-sensor actuated automatic dripirrigation of cotton. In: Proceedings International Conference on Evapotranspiration and Irrigation Scheduling, November, San Antonio, TX, pp. 886-892.

Phene, C.J., Howell, T.A., 1984. Soil sensor control of high-frequency irrigation sys-tems. Trans. ASAE 27 (2), 392-396.

Rodríguez Díaz, J.A., Weatherhead, E.K., Knox, J.W., Camacho, E., 2007. Climatechange impacts on irrigation water requirements in the Guadalquivir river basinin Spain. Reg. Environ. Change 7, 149-159.

Rossman, L.A. (2000). "EPANET: User Manual. Risk Reduction Engineering Laboratory Office of Research and Development". United States Environmental Protection Agency. Cincinnati, $\mathrm{OH}$. 
Shannon, E.L., McDougall, A., Kelsey, K., Hussey, B., 1996. Watercheck-a coordinatedextension program for improving irrigation efficiency on Australian cane farms.In: Proc. Aust. Soc. Sugar Cane Technol, pp. 113-118, 18.

Smith, R.J., Baillie, J.N., McCarthy, A.C., Raine, S.R., Baillie, C.P., 2010. Review ofPrecision Irrigation Technologies and their Application. National Centre of Engi-neering in Agriculture, University of Southern Queensland, Toowoomba NCEAPublication $1003017 / 1$.

Wanjura, D.F., Upchurch, D.R., Mahan, J.R., 2004. Establishing differential irriga-tion levels using temperature-time thresholds. Appl. Eng. Agric. 20 (2), 201-206.

WWF. (2009) Manual de buenas prácticas de riego. Propuestas de WWF para un uso eficiente del agua en la agricultura. Viñedo, Olivar, Cítricos y Fresa. Madrid. Spain. 